Schmerz 2022 · 36:182-188

https://doi.org/10.1007/s00482-021-00614-w

Angenommen: 4. November 2021

Online publiziert: 23. Dezember 2021

๑) Der/die Autor(en) 2021

\title{
Vom Bauchgefühl zum viszeralen Schmerz
}

\section{Effekte negativer Erwartungen im Kontext der Darm-Gehirn- Achse}

\author{
Jana Aulenkamp ${ }^{1,2} \cdot$ Kathrin Steinmüller $^{1} \cdot$ Adriane Icenhour $^{1,3} \cdot$ Sigrid Elsenbruch $^{1,3}$ \\ ' Klinik für Neurologie, Universitätsklinikum Essen, Essen, Deutschland \\ ${ }^{2}$ Klinik für Anästhesiologie und Intensivmedizin, Universitätsklinikum Essen, Essen, Deutschland \\ ${ }^{3}$ Institut für Medizinische Psychologie und Medizinische Soziologie, Ruhr Universität Bochum, Bochum, \\ Deutschland
}

\section{Zusammenfassung}

Störungen der Darm-Gehirn-Achse sind durch komplexe Dysfunktionen auf peripherer und zentralnervöser Ebene gekennzeichnet, die zu viszeraler Hypervigilanz und Hyperalgesie beitragen können und viszeralen Schmerz prägen. An der viszeralen Schmerzmodulation sind zahlreiche kognitive, emotionale und psychoneurobiologische Faktoren beteiligt, die im psychosozialen Therapiekontext das viszerale Schmerzerleben sowohl positiv als auch negativ beeinflussen können. Durch negative Erwartungen vermittelte Noceboeffekte sind bei akuten, aber insbesondere auch bei chronischen viszeralen Schmerzen von hoher klinischer Relevanz; die ihnen zugrunde liegenden Mechanismen sind jedoch bislang unzureichend verstanden. Zur Entstehung und Aufrechterhaltung negativer Erwartungseffekte tragen insbesondere verbale Instruktionen, Vorerfahrungen und Lernprozesse sowie emotionale Faktoren wie Angst und Stress bei. Gezielte Kommunikationsstrategien, ein sensibler Umgang in der Aufklärung und positive Umgebungsbedingungen können in der klinischen Praxis dazu beitragen, ein adäquates Erwartungsmanagement zu etablieren und negative Erwartungseffekte zu minimieren. Zugleich sind translationale Forschungsansätze erforderlich, um tiefere Erkenntnisse bezüglich der Mediatoren und Moderatoren negativer Erwartungseffekte zu erlangen und diese in die Klinik zu übertragen. So kann die Versorgung von Patienten mit Störungen der Darm-Gehirn-Kommunikation verbessert werden.

\section{Schlüsselwörter}

Noceboeffekt · Chronischer Schmerz · Hypervigilanz · Schmerzbezogene Furcht · Psychologische Faktoren

Die Relevanz von Erwartungseffekten für das Schmerzerleben und die Schmerzbewertung wird zunehmend gewürdigt. Während positive Erwartungseffekte in Forschung wie klinischer Praxis vermehrt Beachtung finden, bleiben die Mechanismen und Konsequenzen negativer Erwartungen weniger gut verstanden. Verfügbare Erkenntnisse stammen zudem meist aus der Erforschung somatischer Schmerzen und sind im Kontext viszeraler Schmerzen trotz profunder Effekte auf die Darm-Gehirn-Achse unzureichend. Ein tieferes Verständnis von
Noceboeffekten in der Pathophysiologie viszeraler Schmerzen kann dazu beitragen, negativen Behandlungserwartungen entgegenzuwirken.

In der Alltagssprache häufig als „Bauchgefühle" bezeichnete Wahrnehmungen umfassen ein großes Spektrum, das von eher als intuitiv empfundenen Gefühlen über diffuse Empfindungen bis hin zu bedrohlich erscheinenden viszeralen Schmerzen reicht. Die neurobiologischen Grundlagen und klinischen Implikationen dieses als Viszerozeption bezeichne- 
ten Teilbereichs der Interozeption finden dank neuer Erkenntnisse über die vielfältigen Verbindungen zwischen Darm und Gehirn zunehmend wissenschaftliche Aufmerksamkeit. Interdisziplinäre, translationale Forschungsansätze zur DarmGehirn-Achse an der Schnittstelle zwischen Neurogastroenterologie, Schmerzforschung und Neurowissenschaften liefern in diesem Kontext hochrelevante Erkenntnisse bezüglich normaler sowie gestörter Darm-Gehirn-Interaktionen und der Pathophysiologie und Therapie viszeraler Schmerzen. Unter Würdigung eines biopsychosozialen Erkrankungskonzepts ebnet dies den Weg für einen ganzheitlichen Blick auf die Patienten und schafft damit verbesserte Grundlagen für eine personalisierte Therapie.

\section{》) Negative Erwartungen als Basis für Noceboeffekte sind wissenschaftlich und klinisch unzureichend verstanden}

Das Wissen um Erwartungseffekte innerhalb des psychosozialen Behandlungskontexts spezifisch für den viszeralen Schmerz eröffnet ein zukunftsweisendes und spannendes Forschungsfeld, insbesondere für Störungen der Darm-Gehirn-Interaktion wie das Reizdarmsyndrom. Die Bedeutung positiver Therapieerwartungen, gemeinhin als Placeboeffekte bezeichnet, ist dabei bereits vergleichsweise gut untersucht, sowohl in randomisierten, kontrollierten klinischen Studien verschiedener mit Schmerz und viszeralen Beschwerden einhergehender gastrointestinaler Erkrankungen als auch im Hinblick auf das therapeutische Potenzial eines gezielten Einsatzes von Placebointerventionen in der Behandlung von Patienten mit chronischem viszeralem Schmerz [7]. Negative Erwartungen als Grundlage für Noceboeffekte sind hingegen weder wissenschaftlich noch klinisch hinreichend verstanden, und ein Großteil des Wissens bezüglich der Effekte negativer Erwartungen basiert bislang auf Forschungsarbeiten aus der somatischen Schmerzforschung. Ziel dieser Übersichtsarbeit ist es, für den Bereich der viszeralen Schmerzen im Kontext der Darm-Gehirn-Achse aktuelles Wissen und klinische Implikationen bezüglich negativer Erwartungseffekte zusammen- zufassen, Forschungslücken aufzuzeigen und Perspektiven für die Translation in die Klinik zu eröffnen.

\section{Viszeraler Schmerz und die Darm- Gehirn-Achse}

Interozeptive, viszerale Schmerzen gehen von den inneren Organen aus und unterscheiden sich qualitativ in vielerlei Hinsicht von exterozeptiven, somatischen Schmerzen. Sie sind nur schwer eindeutig zu lokalisieren, werden häufig von neurovegetativen Symptomen wie Übelkeit, Erbrechen, Schwitzen oder erhöhter Herzrate begleitet und können auf angrenzende Körperareale übertragen werden [1]. Zudem werden sie bereits bei vergleichsweise niedriger Intensität in ihrer emotionalen Qualität auch von Gesunden als unangenehmer empfunden und lösen in stärkerem Maße emotionale Reaktionen wie Furcht aus [14], was die hohe Relevanz psychologischer Faktoren im Kontext des viszeralen Schmerzgeschehens unterstreicht.

\section{॥) Die Kommunikation zwischen Darm und Gehirn verläuft bidirektional}

Interessanterweise dokumentieren Hirnbildgebungsstudien, dass die zentralnervösen Prozesse der akuten viszeralen Schmerzwahrnehmung trotz erwartbarer Überlappungen zu anderen Schmerzreizen, insbesondere zu somatischen Schmerzen, aber auch zu nichtnozizeptiven aversiven Stimuli, einzigartige neurale Aktivierungsmuster aufweisen [14, 15, 24]. Vor allem Regionen des Salienznetzwerks, eines neuronalen Netzwerks, das am Erkennen bedeutsamer Reize und an der Integration sensorischer mit emotionalen und kognitiven Informationen beteiligt ist, sind an der Verarbeitung viszeraler Reize stärker beteiligt [11]. Eine mögliche Spezifität der Schmerzverarbeitung und -modulation in Abhängigkeit von der Schmerzlokalisation ist zwar bei Personengruppen mit chronischen Schmerzen noch nicht vergleichend untersucht, es ist jedoch davon auszugehen, dass zumindest ein Teil der Befunde innerhalb spezifischer Gruppen (z.B. Rückenschmerzen) nicht in Gänze auf die viszerale Modalität (z.B. Reizdarmbeschwerden) übertragbar ist.
Grundlage für die viszerale Perzeption und den viszeralen Schmerz ist die Darm-Gehirn-Achse. Die Kommunikation zwischen Darm und Gehirn verläuft bidirektional, wobei neben neuronalen Signalwegen das autonome Nervensystem (primär über den Vagusnerv) sowie neuroendokrine und immunologischeSysteme und das Mikrobiom des Darms eine Rolle spielen [17]. Viszerale Wahrnehmungsprozesse werden sowohl direkt im Gehirn durch kognitive und affektive Mechanismen als auch durch absteigende modulatorische Signalwege beeinflusst, wodurch wiederum die Aktivität des Verdauungstrakts verändert werden kann [17]. Bei einer Dysregulation des Darm-Gehirn-Systems kann es daher zu Hypersensitivität und Hyperalgesie in der Wahrnehmung von physiologischen Signalen kommen, was wiederum eine erhöhte Aufmerksamkeit auf viszerale Reize (Hypervigilanz) und Veränderungen der Magen-Darm-Motilität zur Folge haben kann $[5,17]$. Dies kann zu Störungen führen, die durch chronische viszerale Schmerzen und veränderte Verdauungsfunktionen charakterisiert sind.

\section{Chronischer viszeraler Schmerz bei Störungen der Darm-Gehirn-Achse}

Die Ursachen für chronische viszerale Schmerzen können vielfältig sein. Andauernde bzw. wiederkehrende viszerale Symptome treten häufig auch ohne eindeutig identifizierbare organische Ursache auf. In diesen Fällen spricht man von funktionellen Magen-Darm-Erkrankungen oder in jüngster Zeit von Störungen der Darm-Gehirn-Interaktion, unter denen das Reizdarmsyndrom die häufigste ist [5]. Für Betroffene bedeuten chronische viszerale Beschwerden, insbesondere der viszerale Schmerz, nicht nur erhebliche Beeinträchtigungen des Alltags und eine Reduktion der Lebensqualität. Es ergeben sich auch Herausforderungen für das Gesundheitssystem als Ganzes sowie für die individuelle Patientenführung und ArztPatienten-Kommunikation, bedingt durch eine vermehrte Inanspruchnahme medizinischer Leistungen wie beispielsweise wiederholte Diagnostik und mangelnde Adhärenz, aber auch durch eine hohe Komorbidität mit Affektstörungen [5]. Dies erfordert ein differenziertes diagnosti- 
sches und therapeutisches Vorgehen unter Berücksichtigung eines biopsychosozialen Krankheitsmodells und des Wissens über die komplexen Mechanismen der DarmGehirn-Achse, insbesondere bezüglich der Rolle psychologischer Einflussfaktoren.

Stress ist ein zentraler psychologischer Einflussfaktor, der im Kontext viszeraler Schmerzen und insbesondere im Zusammenhang mit Reizdarmbeschwerden bereits seit vielen Jahren starke Beachtung findet [16]. Diverse Kommunikationswege der Darm-Gehirn-Achse sind an den engen Zusammenhängen zwischen Stress und viszeraler Symptomatik beteiligt, einschließlich des autonomen Nervensystems, des neuroendokrinen und des Immunsystems sowie des Mikrobioms [16]. So kann Stress nachweislich einerseits zu mikrobiellen Veränderungen führen, andererseits lassen sich durch eine Beeinflussung des Mikrobioms, beispielsweise durch Behandlung mit einem Probiotikum, Stressreaktionen sowie abdominelle Störungen modulieren, was insgesamt die Bidirektionalität der Darm-GehirnAchse unterstreicht [16]. Diese Erkenntnisse haben einen Paradigmenwechsel in Bezug darauf eingeleitet, wie funktionelle Magen-Darm-Erkrankungen konzeptualisiert werden, nämlich von einer rein peripheren Störung der Darmmotilität ohne organische Marker zu einer Erkrankung, die durch komplexe Dysfunktionen der Darm-Gehirn-Interaktion mit peripheren und zentralnervösen Komponenten gekennzeichnet ist [17].

\section{》) Dysfunktionale Kognitionen können zur Aufrechterhaltung und Verschlimmerung von Symptomen beitragen}

Neben Stress spielen auch maladaptive Kognitionen eine wichtige Rolle [12]. So können bei Patienten mit Reizdarmsyndrom dysfunktionale Kognitionen wie selektive Aufmerksamkeit und Katastrophisieren in Reaktion auf viszerale Signale zur Aufrechterhaltung und Verschlimmerung von Symptomen beitragen und sind mit der Symptomschwere assoziiert. Auch Furcht, negative Vorerfahrungen und affektive Störungen mit Symptomen der Angst und Depression stehen in direktem Zusammenhang nicht nur mit maladaptiven Kognitionen, sondern auch mit einer veränderten viszeralen Schmerzwahrnehmung [12]. Es ist daher von zentraler Bedeutung, auch diese Faktoren der Schmerzmodulation in den Blick zu nehmen, die, wie im Folgenden erläutert, vermutlich essenziell auch an negativen Erwartungseffekten beteiligt sind.

\section{Erwartungseffekte im psycho- sozialen Behandlungskontext}

Im psychosozialen Behandlungskontext können Erwartungen die Symptom- bzw. Schmerzwahrnehmung sowie das Erleben und Erinnern belastender Symptome stark beeinflussen. Für den Bereich der gastrointestinalen Erkrankungen liegen inzwischen zahlreiche randomisierte, kontrollierte Studien vor, die analog zu vielen anderen Erkrankungen die Bedeutung positiver Therapieerwartungen unterstreichen [7]. Eine aktuelle klinische Studie bei Patienten mit Reizdarmsyndrom erweitert die Datenlage und dokumentiert eine substanzielle Symptomverbesserung nach der Gabe von Placebotabletten, unabhängig davon, ob die Teilnehmer von einem pharmakologischen Wirkstoff ausgingen oder explizit darüber aufgeklärt wurden, dass es sich um wirkstofffreie Placebos handelte (Open-label-PlaceboBehandlung; [19]).

Im Gegensatz zu solchen Effekten positiver Therapieerwartung rückt die Bedeutung negativer Erwartungen und somit potenziell schmerzamplifizierender Noceboeffekte erst langsam in den Fokus systematischer Untersuchungen, obgleich diese im Behandlungskontext höchst relevant sind und den Behandlungsverlauf und -erfolg maßgeblich beeinflussen können. Daten aus den an die Viszeralmedizin angrenzenden Disziplinen zeigen, dass schätzungsweise $40-100 \%$ der Nebenwirkungen von Medikamenten nicht auf den Wirkstoff selbst, sondern auf den Behandlungskontext zurückzuführen sind [20]. Negative Erwartungseffekte können zudem die Wirksamkeit therapeutischer Interventionen beeinträchtigen [3]. Für den viszeralen Schmerz konnte bereits 1987 dokumentiert werden, dass die Beschreibung möglicher gastrointestinaler Nebenwirkungen während des Einwilligungsgesprächs zu einem 6fachen Anstieg der berichteten gastrointestinalen Nebenwirkungen führte [23]. Da "Nocebo" ursprünglich jedoch vor allem unerwünschte Ereignisse beschrieb, die von Studienteilnehmern nach Gabe eines Placebopräparats berichtet wurden [7], und sehr frühe experimentelle Nachweise die Begrifflichkeit „Nocebo" noch nicht verwendeten (für einen kurzen historischen Abriss siehe [7]), wurde der Einfluss negativer Erwartung, insbesondere aber auch seine klinischen Implikationen, möglicherweise in der Wissenschaft und öffentlichen Wahrnehmung lange unterschätzt. In einer neueren prospektiven Studie sagten jedoch einzig psychosoziale Faktoren, vor allem die Erwartung eines langwierigen und komplikationsbehafteten Heilungsprozesses, die Entwicklung eines Reizdarmsyndroms nach einem gynäkologischen Eingriff vorher [30]. Seither wächst auch das Interesse an psychologischen Mechanismen, die der Entstehung und Aufrechterhaltung negativer Erwartungseffekte bei viszeralen Schmerzen zugrunde liegen.

\section{Psychologische Mechanismen negativer Erwartungseffekte}

Aktuelle konzeptionelle Ansätze gehen von verschiedenen, aber nicht zwingend unabhängigen psychologischen Mechanismen aus, die Noceboeffekten zugrunde liegen (• Abb. 1). Dazu gehören insbesondere Faktoren des Behandlungskontexts, vor allem die durch Behandler vermittelten Informationen und Instruktionen sowie individuelle Therapieerfahrungen und Lernprozesse. Laut einer Metaanalyse scheint besonders die Kombination von verbaler Instruktion und Lernprozessen starke hyperalgetische Effekte induzieren zu können [26], wobei dies bislang nicht speziell für viszerale Schmerzen nachgewiesen werden konnte [8].

An einem experimentellen viszeralen Schmerzmodell wurde beobachtet, dass das Erleben und die neuronale Verarbeitung viszeraler Schmerzen allein durch verbale Informationen negativ beeinflussbar sind [28, 29]. Dabei wurde bei gesunden Probanden eine negative Behandlungserwartung durch die Information induziert, einen den Akutschmerz 


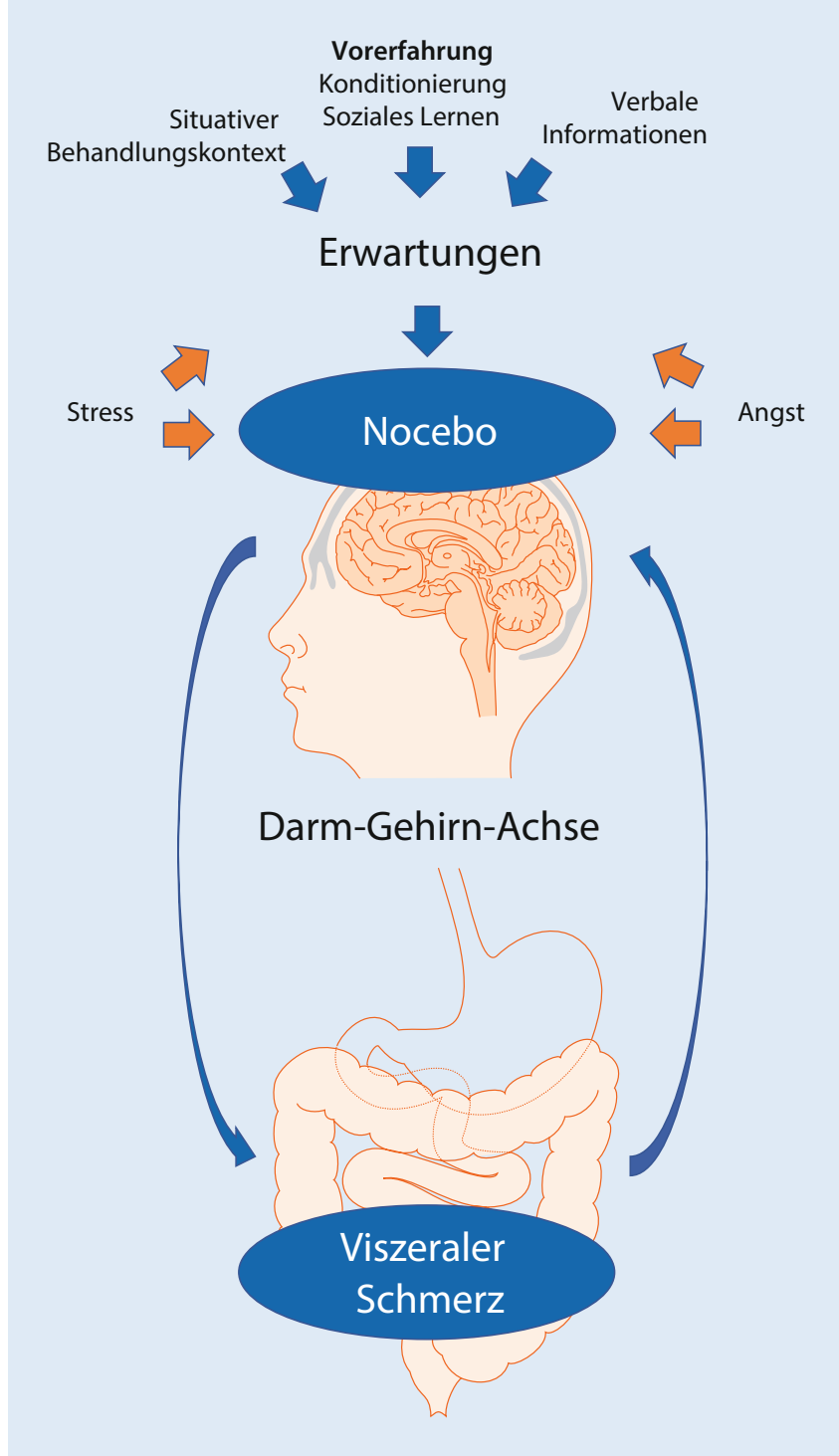

men von Furcht-Vermeidungs-Modellen des chronischen Schmerzes - fundamental an der Aufrechterhaltung von Schmerzen beteiligt [22]. Experimentelle Arbeiten zu viszeralen schmerzbezogenen Lernprozessen bei Gesunden und Patienten mit Reizdarmsyndrom dokumentieren systematisch den Einfluss klassischer Konditionierung auf Erwartungseffekte im Kontext der Darm-Gehirn-Achse [6]. Durch klassische Konditionierung induzierte Veränderungen der Schmerzantizipation können dabei die zentralnervöse Verarbeitung viszeraler schmerzhafter, aber auch nichtschmerzhafter Symptome verändern, insbesondere wenn diese als unvorhersehbar erlebt werden [6], das heißt, wenn es keine spezifischen Hinweise gibt, die das Auftreten von Schmerzen vorhersagen, oder kein Zusammenhang zwischen spezifischen Hinweisen und Schmerzen erkannt wird [22], was experimentell anhand von Bewertungen der Kontingenz zwischen prädiktiven Reizen und Schmerzstimuli quantifiziert werden kann.

\section{\) Frühere Schmerzerfahrungen und -behandlungen tragen zu negativen Erwartungseffekten bei}

Im Bereich somatischer Schmerzen ist gut belegt, dass Unvorhersehbarkeit Hyperalgesie fördern kann [22], sodass dies auch bei Patienten mit viszeraler Symptomatik, die oft mit unvorhersehbaren Schmerzen konfrontiert sind, zu einer Verschlimmerung ihrer Symptome beitragen könnte. Interessant sind in diesem Zusammenhang auch erste Belege dafür, dass akuter Stress negative Erwartungseffekte bei Gesunden offenbar verstärkt [28]. Da sowohl die viszerale Schmerzsensitivität als auch das differenzielle schmerzbezogene Lernen durch das Stresshormon Kortisol modifizierbar sind [2] und der Erwerb schmerzbezogener Furcht bei Patienten mit Reizdarmsyndrom verändert ist [10], erscheinen psychologische Faktoren wie Stress und (erlernte) Furcht als Amplifikatoren des Noceboeffekts insbesondere in Bezug auf viszerale Schmerzen naheliegend. Aktuelle Daten zeigen zudem, dass eine Aktivierung des zentralnervösen Furchtnetzwerks auch durch harmlose interozeptive „Bauchgefühle“ evoziert werden 
kann, wenn diese als erlernte Prädiktoren viszeraler Schmerzen wahrgenommen werden [11]. Bei derartigen interozeptiven Lern- und Gedächtnisprozessen scheinen kontextuelle Faktoren und Veränderungen der Aufmerksamkeit maßgeblich beteiligt zu sein. Diese Befunde unterstreichen die Relevanzinterozeptiver Konditionierung in der Pathophysiologie viszeraler Schmerzen mit klaren Implikationen für negative Erwartungseffekte im Behandlungskontext, bei denen interozeptiver Hypervigilanz eine zentrale Rolle zukommen könnte. Insgesamt bilden Modelle schmerzbezogener Lern- und Gedächtnisprozesse wertvolle translationale Ansätze und liefern in Form des Extinktionslernens auch die konzeptionelle Basis für expositionstherapeutische Ansätze bei chronischem viszeralem Schmerz, die Furcht und negative Erwartungen reduzieren und positive Effekte auf die Schmerzsymptomatik haben können [6].

\section{Implikationen für die Therapie chronischer viszeraler Schmerzen}

Aus Erkenntnissen über negative Erwartungseffekte lassen sich verschiedene Implikationen für die Optimierung der Behandlung von Patienten mit chronischen viszeralen Schmerzen ableiten. Dabei sollten der Behandlungskontext, die Kommunikation und modulierende psychologische Faktoren gleichermaßen in den Fokus genommen werden. Patienten mit chronischen viszeralen Schmerzen haben oft negative Vorerfahrungen im Behandlungskontext gemacht, etwa in der Kommunikation oder mit früheren Behandlungsversuchen. Es liegt nahe, dass gerade bei sogenannten Ausschlussdiagnosen wie dem Reizdarmsyndrom, für das nach teils aufwendiger Diagnostik zudem keine eindeutige, erfolgreiche Therapiestrategie existiert, das Risiko für negative Vorerfahrungen besonders hoch ist. Dadurch geprägte Erwartungen und Ängste im Vorfeld zu identifizieren, kann dazu beitragen, die Arzt-Patienten-Beziehung zu optimieren und durch frühere negative Erfahrungen induzierten Noceboeffekten entgegenzuwirken [13]. Neben einer auf den Patienten abgestimmten Aufklärung über den Einfluss von Noceboeffekten im Behandlungskontext [9] ermöglichen multimodale An- sätze in der Schmerztherapie es, Stress, erlernte Furcht, maladaptive Kognitionen und Vermeidungsstrategien als Amplifikatoren negativer Erwartungseffekte in den Fokus zu rücken [22] und adäquate Bewältigungsstrategien zu vermitteln, um Noceboeffekte effizient zu reduzieren [21].

\section{I) Eine verbesserte Kommunikation kann die Patientenerwartung positiv beeinflussen}

Erkrankungsübergreifende Konzepte machen deutlich, dass die Patientenerwartung durch eine verbesserte Kommunikation seitens aller am Behandlungsprozess Beteiligten positiv beeinflusst werden kann [21]. So kann zur Vermeidung negativer Erwartungseffekte die informierte Einwilligung angepasst werden, unter anderem lassen sich Angaben zu Nebenwirkungen positiv formulieren (Beispiel: „95\% der Betroffenen haben keine Probleme ..."; [27]). Ein Verzicht auf Informationen über Nebenwirkungen erzielt zwar positive Ergebnisse, muss allerdings ethisch und berufsrechtlich diskutiert werden [31]. Neue Wege zur Vermeidung negativer Erwartungseffekte unter Beibehaltung der informierten Zustimmung werden derzeit erarbeitet [31]. Eine gezielte Schulung und Sensibilisierung medizinischen Personals sowohl zur Relevanz von Erwartungseffekten als auch zur Vermeidung von Noceboeffekten wird als zentraler Faktor betrachtet [9]. Dabei sollten nicht nur die verbale Kommunikation, sondern auch nonverbale Verhaltensweisen wie Blickkontakt und empathische Zuwendung Berücksichtigung finden, die sich nachweislich auf das Schmerzerleben auswirken können [4]. Zentral und bereits systematisch integriert [18] ist die Anerkennung, dass eine Optimierung der Arzt-Patienten-Kommunikation insbesondere bei Patienten mit Störungen der Darm-Gehirn-Interaktion eine tragende Säule jeglicher Diagnostik und Therapie sein sollte.

\section{Fazit für die Praxis}

- Negative Erwartungseffekte beim viszeralen Schmerz können durch verbale Instruktionen, den Behandlungskontext und Lernprozesse induziert und moduliert werden.
- Psychologische Faktoren wie maladaptive Kognitionen, Angst und Stress sind essenziell an negativen Erwartungseffekten beteiligt und können sich auch direkt auf die viszerale Symptomatik auswirken.

- Bei der Gestaltung einer tragfähigen und vertrauensvollen Arzt-Patienten-Beziehung und zur Reduktion negativer Erwartungseffekte sollten im Rahmen multimodaler Ansätze der Behandlungskontext, die Kommunikation und modulierende psychologische Faktoren gleichermaßen in den Fokus genommen werden.

- Eine Optimierung der Arzt-PatientenKommunikation, insbesondere bei Patienten mit Störungen der Darm-GehirnInteraktion, sollte eine tragende Säule jeglicher Diagnostik und Therapie sein.

Korrespondenzadresse

\section{Jana Aulenkamp}

Klinik für Anästhesiologie und Intensivmedizin, Universitätsklinikum Essen

Hufelandstraße 55, 45147 Essen, Deutschland jana.aulenkamp@uk-essen.de

Förderung. Diese Arbeit wurde gefördert durch die Deutsche Forschungsgemeinschaft (DFG) - Projektnummer 422744262 - TRR 289.

Funding. Open Access funding enabled and organized by Projekt DEAL.

\section{Einhaltung ethischer Richtlinien}

Interessenkonflikt. J. Aulenkamp, K. Steinmüller A. Icenhour und S. Elsenbruch geben an, dass kein Interessenkonflikt besteht.

Für diesen Beitrag wurden von den Autoren keine Studien an Menschen oder Tieren durchgeführt. Für die aufgeführten Studien gelten die jeweils dort angegebenen ethischen Richtlinien.

Open Access. Dieser Artikel wird unter der Creative Commons Namensnennung 4.0 International Lizenz veröffentlicht, welche die Nutzung, Vervielfältigung, Bearbeitung, Verbreitung und Wiedergabe in jeglichem Medium und Format erlaubt, sofern Sie den/die ursprünglichen Autor(en) und die Quelle ordnungsgemäß nennen, einen Link zur Creative Commons Lizenz beifügen und angeben, ob Änderungen vorgenommen wurden.

Die in diesem Artikel enthaltenen Bilder und sonstiges Drittmaterial unterliegen ebenfalls der genannten Creative Commons Lizenz, sofern sich aus der Abbildungslegende nichts anderes ergibt. Sofern das betreffende Material nicht unter der genannten Creative Commons Lizenz steht und die betreffende Handlung nicht nach gesetzlichen Vorschriften erlaubt ist, ist für die oben aufgeführten Weiterverwendungen des Materials die Einwilligung des jeweiligen Rechteinhabers einzuholen. 
Weitere Details zur Lizenz entnehmen Sie bitte der Lizenzinformation auf http://creativecommons.org/ licenses/by/4.0/deed.de.

\section{Literatur}

1. Aziz Q, Giamberardino MA, Barke A et al (2019) The IASP classification of chronic pain for ICD-11: chronic secondary visceral pain. Pain 160(1):69-76

2. Benson S, Siebert C, Koenen LR et al (2019) Cortisol affects pain sensitivity and pain-related emotional learning in experimental visceral but not somatic pain: a randomized controlled study in healthy men and women. Pain 160(8):1719-1728

3. Bingel U, Wanigasekera V, Wiech K et al (2011) The effect of treatment expectation on drug efficacy: imaging the analgesic benefit of the opioid remifentanil. Sci Transl Med 3(70):70ra14

4. DanialiH,Flaten MA (2019)A qualitative systematic review of effects of provider characteristics and nonverbal behavior on pain, and placebo and nocebo effects. Front Psychiatry 10:242

5. Drossman DA, Hasler WL (2016) Rome IV-functional GI disorders: disorders of gut-brain interaction. Gastroenterology 150(6):1257-1261

6. Elsenbruch S, Benson S, Ricarda Koenen L et al (2020) From gut feelings to memories of visceral pain. Neuroforum 26(3):171-177

7. Elsenbruch S, Enck P (2015) Placebo effects and their determinants in gastrointestinal disorders. Nat Rev Gastroenterol Hepatol 12(8):472-485

8. Elsenbruch S, Labrenz F (2018) Nocebo effects and experimental models in visceral pain, 1st ed. Int Rev Neurobiol 138:285-306

9. Evers AWM, Colloca L, Blease C et al (2021) What should clinicians tell patients about placebo and nocebo effects? Practical considerations based on expert consensus. Psychother Psychosom 90(1):49-56

10. Icenhour A, Langhorst J, Benson S et al (2015) Neural circuitry of abdominal pain-related fear learning and reinstatement in irritable bowel syndrome. Neurogastroenterol Motil 27(1):114-127

11. Icenhour A, Petrakova L, Hazzan N et al (2021) When gut feelings teach the brain to fear pain: context-dependent activation of the central fear network in a novel interoceptive conditioning paradigm. Neuroimage 238:118229

12. Kennedy PJ, Clarke G, Quigley EM, Groeger JA, Dinan TG, Cryan JF (2012) Gut memories: towards a cognitive neurobiology of irritable bowel syndrome. Neurosci Biobehav Rev 36(1):310-340

13. Klinger R, Blasini M, Schmitz J, Colloca L (2017) Nocebo effects in clinical studies: hints for pain therapy. Pain Rep 2(2):e586

14. Koenen LR, Icenhour A, Forkmann K et al (2017) Greater fear of visceral pain contributes to differences between visceral and somatic pain in healthy women. Pain 158(8):1599-1608

15. Koenen LR, Pawlik RJ, Icenhour A et al (2021) Associative learning and extinction of conditioned threat predictors across sensory modalities. Commun Biol 4:553

16. Labanski A, Langhorst J, Engler $\mathrm{H}$, Elsenbruch $\mathrm{S}$ (2020) Stress and the brain-gut axis in functional and chronic-inflammatory gastrointestinal diseases: a transdisciplinary challenge. Psychoneuroendocrinology 111:104501

17. Labus JS, Tun G, Kilpatrick LA, Rao SSC, Mayer EA, Tillisch K (2019) Neuroimaging and biomarkers in functional gastrointestinal disorders: what the scientists and clinicians need to know about

\section{From gut feeling to visceral pain. Effects of negative expectations in the context of the gut-brain axis}

Disturbances of the gut-brain axis are characterized by complex dysfunctions on peripheral and central nervous system levels, which can contribute to visceral hypervigilance and hyperalgesia and imprint visceral pain. Numerous cognitive, emotional and psychoneurobiological factors are involved in visceral pain modulation, which in the psychosocial treatment concept can have a positive as well as a negative impact on the experience of visceral pain. Nocebo effects induced by negative expectations are of high clinical relevance in acute and especially in chronic visceral pain but the underlying mechanisms remain insufficiently understood. Verbal instructions, previous experiences and learning processes as well as emotional factors, such as fear and stress contribute to the development and maintenance of negative expectation effects. Targeted communication strategies, a sensitive use of information in the clarification and positive environmental context conditions can contribute to establishing an adequate expectation management and minimize negative expectation effects in the clinical practice. At the same time, translational research approaches are required to gain further insights into the mediators and moderators of negative expectation effects and to transfer these into clinical practice. In this way the treatment of patients with disorders of the gut-brain communication can be improved.

\section{Keywords}

Nocebo effect · Chronic pain · Hypervigilance $\cdot$ Fear, pain-related $\cdot$ Psychological factors

basic neuroimaging, biomarkers, microbiome, gut and brain interactions. In: Clinical and basic neurogastroenterology and motility. Elsevier, S31-61

18. Layer $\mathrm{P}$, Andresen V, Allescher $\mathrm{H}$ et al (2021) Update S3-Leitlinie Reizdarmsyndrom: Definition, Pathophysiologie, Diagnostik und Therapie des Reizdarmsyndroms der Deutschen Gesellschaft für Gastroenterologie, Verdauungs- und Stoffwechselkrankheiten. DGVS Deutsche Gesellschaft für Gastroenterologie, Verdauungs- und Stoffwechselkrankheiten,

19. Lembo A, Kelley JM, Nee J et al (2021) Openlabel placebo vs double-blind placebo for irritable bowel syndrome: a randomized clinical trial. Pain 162(9):2428-2435

20. Mahr A, Golmard C, Pham E, lordache L, Deville L, Faure $P$ (2017) Types, frequencies, and burden of nonspecific adverse events of drugs: analysis of randomized placebo-controlled clinical trials. Pharmacoepidemiol Drug Saf 26(7):731-741

21. Manaï $M$, van Middendorp $H$, Veldhuijzen DS, Huizinga TWJ, Evers AWM (2019) How to prevent, minimize, or extinguish nocebo effects in pain: a narrative review on mechanisms, predictors, and interventions. Pain Rep 4(3):e699

22. Meulders A (2020) Fear in the context of pain: lessons learned from 100 years of fear conditioning research. Behav Res Ther 131:103635

23. Myers MG, Cairns JA, Singer J (1987) The consent form as a possible cause of side effects. Int J Clin Pharmacol Ther 42(3):250-253

24. Van Oudenhove L, Kragel PA, Dupont P et al (2020) Common and distinct neural representations of aversive somatic and visceral stimulation in healthy individuals. Nat Commun 11(1):5939

25. Peerdeman KJ, Van Laarhoven AIM, Keij SM et al (2016) Relieving patients' pain with expectation interventions: a meta-analysis. Pain 157(6):1179-1191
26. Petersen GL, Finnerup NB, Colloca L et al (2014) The magnitude of nocebo effects in pain: a metaanalysis. Pain 155(8):1426-1434

27. Petrie KJ, RiefW (2019) Psychobiological mechanisms of placebo and nocebo effects: pathways to improve treatments and reduce side effects. Annu Rev Psychol 70:599-625

28. Roderigo T, Benson S, Schöls M et al (2017) Effects of acute psychological stress on placebo and nocebo responses in a clinically relevant model of visceroception. Pain 158(8):1489-1498

29. Schmid J, Bingel U, Ritter C et al (2015) Neural underpinnings of nocebo hyperalgesia in visceral pain: a fMRI study in healthy volunteers. Neuroimage 120:114-122

30. Sperber AD, Morris CB, Greemberg L et al (2008) Development of abdominal pain and IBS following gynecological surgery: a prospective, controlled study. Gastroenterology 134(1):75-84

31. Webster RK, Rubin GJ (2019) Influencing sideeffects to medicinal treatments: a systematic review of brief psychological interventions. Front Psychiatry 9:775 
Hier steht eine Anzeige.

黑 Springer 